Disaster Medicine and Public

Health Preparedness

www.cambridge.org/dmp

\section{Report from the Field}

Cite this article: Bell SA, Krienke L, Quanstrom K. Alternative care sites during the COVID-19 pandemic: Policy implications for pandemic surge planning. Disaster Med Public Health Prep. doi: https://doi.org/10.1017/ dmp.2021.241.

\section{Keywords:}

alternative care site; pandemic; policy; preparedness

\section{Corresponding Author:}

Sue Anne Bell,

Email: sabell@umich.edu.

\section{Alternative Care Sites During the COVID-19 Pandemic: Policy Implications for Pandemic Surge Planning}

\author{
Sue Anne Bell PhD, FNP-BC, NHDP-BC ${ }^{1}$ (D) Lydia Krienke $\mathrm{BA}^{2}$ and \\ Kathryn Quanstrom $\mathrm{MD}(\mathrm{c})^{3}$
}

${ }^{1}$ University of Michigan School of Nursing, University of Michigan, Ann Arbor, MI, USA; ${ }^{2}$ Johns Hopkins University School of Nursing, Johns Hopkins University, Baltimore, MD, USA and ${ }^{3}$ University of Michigan Medical School, University of Michigan, Ann Arbor, MI, USA

\begin{abstract}
Alternative care sites (ACS) across the United States were widely underutilized during the coronavirus disease (COVID-19) outbreak, while the volume and severity of COVID-19 cases overwhelmed health systems across the United States. The challenges presented by the pandemic have shown the need to design surge capacity principles with consideration for demand that strains multiple response capabilities. We reviewed current policy and previous literature from past ACS as well as highlighted challenges from the COVID-19 pandemic, to make recommendations that can inform future surge capacity planning. Our recommendations include: (1) Preparedness actions need to be continuous and flexible; (2) staffing needs must be met as they arise with solutions that are specific to the pandemic; 3 ) health equity must be a focus of ACS establishment and planning; and (4) ACS should be designed to function without compromising safe and effective care. A critical opportunity exists to identify improvements for future use of ACS in pandemics.
\end{abstract}

\section{Background}

In the spring of 2020, affected cities across the United States rushed to remain ahead of an expected surge of coronavirus disease (COVID-19) patient admissions by rapidly constructing alternative care sites (ACS), also known as field hospitals. ACS, defined as a location converted to provide either inpatient and/or outpatient health care services when existing facilities are compromised by a hazard impact or when an expected volume of patients exceeds available capacity and/or capabilities, ${ }^{1}$ can exist inside or outside of the health systems they are intended to support, such as in convention centers, athletic facilities, and shuttered hospitals. By the end of April 2020, 28 freestanding ACS with capacity ranging from 50 beds to 3000 beds were either under construction or open. ${ }^{1}$ In one of the hardest hit areas of the country, the converted Javits Center in New York City was initially planned for up to 2600 beds. However, at the peak of the pandemic, while nearby hospitals were overwhelmed, only around 1000 patients in total were treated at the Javits Center before its closure. ${ }^{2}$ The TCF Center in Detroit experienced a similar pattern, ${ }^{3}$ drawing questions regarding the utility of the design of these facilities to meet the needs of the types of COVID-19 care they were designed for.

Extensive planning for use of ACS has occurred in the past as part of pandemic preparedness and in the aftermath of other types of disasters, hurricanes being a notable example. However, the complexities around the nationwide, rapid, large-scale application of these facilities during the COVID-19 pandemic caught many by surprise. The Federal Healthcare Resilience Task Force (FHRTF), made up of representatives from the Federal Emergency Management Agency, Army Corps of Engineers, and the Assistant Secretary for Preparedness and Response, outlined recommendations for establishing ACS specific to COVID-19 in a toolkit released in late March of $2020,{ }^{1}$ well after many ACS were already underway. Subsequent updates to the toolkit were released in April and again in June of 2020, and the toolkit is no longer being maintained.

The pandemic has provided an opportunity to re-imagine surge capacity principles with consideration for how capacity can be best supported when demand across regions strains and overwhelms local response capabilities. As hospitals around the world continue to address additional waves of COVID-19 patients and consider the real possibility of future pandemics, learning from the experiences of ACS stakeholders at all levels of coordination during the pandemic is critical to strengthen future responses.

Here, we advance 4 critical recommendations: (1) Preparedness actions must be continuous, nimble, and flexible; (2) staffing needs must be addressed with solutions that are specific to the need; (3) health equity must be a focus of ACS establishment and planning; and (4) ACS should
University Press on behalf of Society for Disaster Medicine and Public Health, Inc.

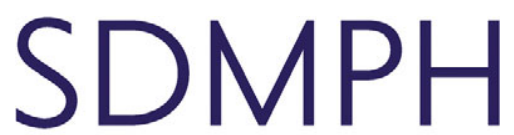

SOCIETY FOR DISASTER MEDICINE \& PUBLIC HEALTH 
be designed to function without compromising safe and effective care. Our recommendations, which are not intended to be allencompassing, stem from review of past ACS literature and policy, as well as insight from the current COVID-19 pandemic.

\section{Preparedness Actions Need to Be Continuous, Nimble, and Flexible}

Optimizing a system's ability to "surge in place" with plans in place for quick and nimble ACS launch will facilitate the ability to uphold occupational safety and standards of care. ${ }^{4}$ Planning needs to be flexible enough to coordinate with efficiency when multiple health care systems within a region are in need of surge support simultaneously. These planning efforts must be ongoing, in order to create an effective safety net before the need becomes overwhelming. Echoing the widely-used statement, all disasters are local, although federal resources are critical in providing support necessary to construct ACS, it is vital that the management, organization, and planning be spearheaded at the local level, and be nimble enough to pivot quickly to meet community needs. As seen with the limitations of the Strategic National Stockpile during the early stages of the pandemic, federal resource allocation may be unavailable to support the needs of jurisdictions due to competing demands.

Planning should consider the worst-case scenario, one that models significant material or human loss, such as was expected with early modeling estimates of COVID-19, without social distancing. Thinking about the 4 "S's" of logistics - staff, stuff, space, and systems - securing equipment, adequate staffing plans, appropriate facilities, and other planning measures should occur in advance as much as possible in order for ACS to reach full utility and potential. As ACS were initially established to cope with large COVID-19 patient volumes in the spring of 2020, extreme shortages of personal protective equipment (PPE) existed, leading to competition for federal funding and resources. Future pandemics may be a reality, making planning for standing up ACS a wide consideration. Assessing locations, doing site planning, and strengthening relationships with emergency managers at the local, state, tribal, territorial and federal levels, alongside planning for needed supplies and staffing resources, is critical to ensuring safe and effective care provisions in the event ACS are urgently needed., ${ }^{1,4}$

\section{Meeting Staffing Needs as They Arise With Solutions That Are Specific to the Pandemic}

During the initial wave of the COVID-19 pandemic in the United States, widespread staffing shortages emerged due to both COVID19 exposure and infection of health care workers and high patient volume. In some areas, non-COVID-related care dramatically dropped, both from health care systems moving to essential services only and widespread avoidance of health care due to fears of being infected, while in others staffing shortages were dire. Badly needed were the highly technical skills of critical care nurses and respiratory therapists, which were focused on providing inpatient, high-acuity care.

The pandemic has made clear the need for staffing models that are created and implemented so that providers and health systems operate in a state of readiness. Leaders in ACS preparation and administration must consider building a self-sufficient staffing model that can operate without support from outside entities. Two critical aspects of staffing are investing in specialized training resources, such as critical care, and developing plans to include task shifting by health care professionals, who can safely take on less complex aspects of clinical care, thereby freeing up staff to focus on care that requires high-level skills. This may support keeping staffing ratios in pace with an exponential increase in patients, as well as distribute the clinical skills needed to provide specific types of care. Strengthening state-organized volunteer registries and the Emergency Management Assistance Compact, a framework for inter-state support, with additional federal support, could be an avenue to address future critical staffing needs. Those standing up ACS should consider how significantly federal staffing resources, such as Disaster Medical Assistance Teams, might be depleted during a time of national need and widespread impact. While these federal resources can provide essential staffing support for some locales, this type of federal response is not scalable to a pandemic that affects the majority of communities in the United States; further, these teams may not have the specialized training specific to the needs of the community. Like all resources, these are finite and limited in the scope of how many practitioners can be deployed at once to multiple institutions in need. In all cases, staff must be supplied with disease-specific training and PPE that will provide adequate protection from infection in accordance with the moral and ethical obligations of health care employers to ensure the safety of their employees. ${ }^{5}$

\section{Health Equity Must Be a Focus of ACS Establishment and Planning}

The COVID-19 pandemic has illustrated that crises most often disproportionally affect those oppressed through policy choices and discourses of racial inferiority in our society. ${ }^{6}$ Development of ACS must be considered within a health equity framework, where planning incorporates an understanding of the ingrained structural and systemic inequalities that communities may face. When designed to focus on targeting inequities, the ACS will have a better chance of providing care that may help alleviate the deep racial and socioeconomic divide seen in disasters, in general, specifically in COVID-19-related morbidity and mortality. The planning of ACS should attempt to remove as many barriers to careseeking as possible, including considering locations and communities that will have the most needs and also the most collective preventable loss. Similarly, preparation efforts must be rooted in the specific needs and values of the communities served by each health care institution. Administrators of health systems may be closer to the community-level than state or federal planners and therefore must be aware of specific needs of the areas they serve. ACS must be structured to consider not only the type and level of care anticipated for ACS to serve the projected needs of a community, but also how the structure of the local health care delivery system augmented with ACS needs to adapt in order to function effectively. ${ }^{2}$

\section{ACS Should Be Designed to Function Without Compromising Safe and Effective Care}

The goal of an effective ACS should be to relieve pressure generated by large numbers of patients in order to allow practitioners to continue to provide care of the highest quality with competency and respect. Early in the pandemic, non-essential services were drastically reduced by many health systems, in order to reserve available resources for COVID-19 care. Health care seeking for nonCOVID-related medical needs dipped 50\% throughout the nation early in the pandemic ${ }^{7}$; in Northern California alone, within the Kaiser Permanente medical system, hospitalizations due to acute conditions such as myocardial infarctions dropped $48 \%$ in the period from January to April 2020 when compared with the same 
months in the year prior. ${ }^{8}$ Additionally, states that took the Centers for Disease Control and Prevention guidelines seriously about physical distancing, mask use, and other measures to decrease COVID-19 cases saw transmission of the disease effectively slowed, resulting in the expected surge of patients to not occur. ${ }^{8,9}$ However, local entities struggled to meet needs without the expected state and federal material and human assets, and competition for resources locally and between states both stalled and limited an effective and safe community response. The rapid scale-up of ACS at multiple locations across the country meant that essential supplies outside of PPE, such as cots, linens, and privacy screens, were difficult or impossible to source, as supply chains were quickly exhausted or restricted by global disruption, and challenges in coordinating public and private stakeholders emerged. In light of these challenges, ACS must be designed to uphold the highest standards of care, and, while this sounds straightforward, the pandemic presented multiple ethical concerns around standards of care in a crisis. ${ }^{10}$ Resources, such as PPE, were sorely limited early in the pandemic while guidelines for use of PPE shifted, seemingly based on supply availability rather than evidence. Thinking again about "Staff, Space, Stuff and Systems," ACS should ensure that they can provide the necessary amount of staff and equipment in an appropriate setting so that patients can continue to receive optimal quality of care, and practitioners can provide care that does not compromise their safety. Given the specialized critical care needs of COVID-19 patients and the challenges of providing this type of care in a temporary environment, the reduction of nonessential health services by partner health systems is an important consideration in deciding to stand up an ACS.

\section{Conclusion}

While the COVID-19 pandemic has caused, and continues to cause, devastating effects across the United States, it has provided a rare opportunity to identify improvements for future use of ACS in pandemics. The 4 recommendations outlined here are required when considering future ACS planning. Now is the time to prepare.
Acknowledgments. The authors acknowledge Nicole Lurie, MD, MPH, for reviewing this manuscript and providing expert input.

Conflict(s) of Interest. The authors declared no potential conflicts of interest with respect to the research, authorship, and/or publication of this paper.

\section{References}

1. Assistant Secretary for Preparedness and Response. Federal Healthcare Resilience Task Force Alternate Care Site (ACS) Toolkit. Washington, DC: US Department of Health and Human Services; 2020.

2. Jada Y, Guarino B. The military's medical "relief valves" remain mostly empty in New York. This is why. Washington, DC: The Washington Post; 2020.

3. LeBlanc B. Michigan: 8 patients admitted so far to TCF Center's 1,000-bed setup. Detroit, MI: The Detroit News; 2020.

4. Hick JL, Christian MD, Sprung CL. Chapter 2. Surge capacity and infrastructure considerations for mass critical care. Recommendations and standard operating procedures for intensive care unit and hospital preparations for an influenza epidemic or mass disaster. Intensive Care Med. 2010; 36(Suppl 1):S11-S20.

5. Venkat A, Wolf L, Geiderman JM, et al. Ethical issues in the response to Ebola virus disease in US emergency departments: a position paper of the American College of Emergency Physicians, the Emergency Nurses Association and the Society for Academic Emergency Medicine. J Emerg Nurs. 2015;41(2):e5-e16.

6. Nixon SA. The coin model of privilege and critical allyship: implications for health. BMC Public Health. 2019;19(1):1637.

7. Wong L, Hawkins J, Langness S, et al. Where are all the patients? Addressing COVID-19 fear to encourage sick patients to seek emergency care. New England Journal of Medicine Catalyst. N Engl J Med. 2020; epub. doi:10.1056/CAT.20.0193.

8. Matrajt L, Leung T. Evaluating the effectiveness of social distancing interventions to delay or flatten the epidemic curve of coronavirus disease. Emerg Infect Dis. 2020;26(8).

9. Bell SA, Dossett LA, Cespero J, et al. T-minus 10 days: the role of an academic medical institution in field hospital planning. Prehosp Disaster Med. 2021;36(3):338-343.

10. Hodge JG Jr, Hanfling D, Hick JL, Piatt JL. Diminishing disparities in U.S. crisis standards of care: medical and legal challenges. Lancet. 2021;34:100838. 